\title{
Book Review: Data Feminism
}

\section{Shannon O’Reilly}

\section{Abstract:}

This book review critiques Lauren F. Klein and Catherine D'lgnazio's Data Feminism (2020). Klein and D'lgnazio take a visual approach to provide a synopsis-underpinned by social and political commentary-that explores the avenues through which data science and data ethics shape how contemporary technologies exploit injustices related to race and gender. Klein and D'lgnazio offer examples of this exploitation, such as the discriminatory surveillance apparatus that relies on racial profiling tactics. These examples are emboldened by the use of contemporary data strategies that-on the surface-strive to achieve a more equitable and 'neutral' hierarchal society. This review examines the text's visual approach to demonstrating institutional inequities and the authors' acknowledgement of their own privilege, specifically the role they play in upholding the oppressive systems they seek to dismantle through collaboration and intersectional analysis.

\section{Keywords:}

Book review, data science, feminism, data ethics, intersectionality

DOI:10.33137/ijournal.v6i2.36457

(c) 2021, O'Reilly, S. Book Review: Data Feminism. This is an Open Access article distributed under CC-BY. (a) $\stackrel{(i)}{\text { iv }}$ 
What does the documentation of femicides in Mexico have in common with gender selection options on your Facebook profile? Catherine D'lgnazio and Lauren F. Klein consider these two phenomena to be examples of data utilization that evince the pervasiveness of structural oppressors and hierarchies, such as cissexism and racism, within the realm of data. Data Feminism poses three fundamental questions that ask the reader to contextualize data consumption and production by way of feminist theory: Data science by whom? Data science for whom? Data science with whose interests in mind? By answering these questions, the book succeeds in its aim to emphasize the ways in which intertwined structural powers maintain the status quo and oppress minoritized individuals in a data-driven world, while also espousing techniques to harness this power in order to rectify inequalities.

Data Feminism is organized around seven core principles, with a chapter devoted to each one. These seven chapters seek to succinctly analyze and critique power structures in data infrastructures through visual and written examples of data-driven oppression and the subsequent attempts to confront unjust structures. Throughout the book, D'lgnazio and Klein employ Patricia Hill Collins' model of the matrix of domination and its four subsets - structural, hegemonic, disciplinary, and interpersonal - in order to examine the configurations of power and contexts that disempower those who are most likely to be oppressed and subjugated via inequitable societal data infrastructures.

The authors highlight a performance from Joy Buolamwini in which she reveals the racism in facial detection programs by wearing a white face mask to demonstrate that facial-analysis software does not detect Black faces. This issue resides in the interpersonal domain, but when this domain is exercised within a dominant collective, it penetrates the three other domains. The programmers and software engineers who code the ubiquitous data-driven structures that permeate our everyday lives, whether consensually or non-consensually, tend to be white cisgender heterosexual men. D'lgnazio and Klein acknowledge this representative discrepancy several times throughout each chapter and rightly assert that facial-recognition data systems are codified to be racist - a concept they refer to as 'codified oppression.' What's more, the solution to this problem is paradoxical. Increased data collection of minoritized groups can ameliorate racist algorithms through inclusivity, while simultaneously making these groups more vulnerable to state surveillance, over-policing, and incarceration.

Data Feminism vehemently rejects the notion of universal objectivity during the data visualization process. Donna Haraway's 'god trick' theory that contemplates the idea that the viewer has the ability to neutrally visualize data by adopting an illusory objective viewpoint, is evoked to iJournal, Vol 6, No. 2, 
challenge the widely held belief that quantitative data analysis is superior to qualitative data analysis. Conversely, data visceralization actively repudiates the absolutism of the 'god trick.' Rather, it embraces the relativism of positionality - a theory that acknowledges the fact that information processing behaviors are inherently influenced by our varying and diverse perspectives, which are determined by culture and context. Data visceralization relies on galvanizing the senses and moving beyond the strictly optical consumption of data. In a performance art piece at the MoMA entitled A Sort of Joy, male and female performers hold iPads and act as stereotypical museum goers while they read the names of artists in the MoMA collection. They follow a list that begins with artists with the most amount of work in the collection. Initially all the names are male-Johns, Roberts, Davids - but eventually, one of the female performers recites the name Mary. This example shows data visceralization in action as the audience feels the data rather than seeing it. The rhetorical data visceralization these types of data performances rely on exploits affect in order to elicit an emotionally driven physiological reaction. Data Feminism considers visual minimalism to not only be a reductive approach to data analysis but an actively harmful and misguided paradigm.

If there's something D'lgnazio and Klein want the reader to know about them, it's the fact that they are white, college educated, middle class women who intrinsically occupy positions of power and privilege, and they are constantly learning and bettering themselves as humans and academics. They actively work to centre people of colour as well as LGBTQ+, Indigenous, and female voices throughout each chapter, even going as far as to include an accountability table in their bibliography that highlights structural problems - such as patriarchy and colonialism - and their aspirational, draft, and final metrics during the research, writing, and peer review process. Their dedication to transparency and accountability is admirable, especially their dedication to centering the Global South - a frequent blind spot in data scholarship. Representation and identity politics only begin to skim the surface of the underlying systemic issues that make centring marginalized voices such a radical act, most notably for white academics who in this text frequently make it a point to highlight their efforts to do so.

D'lgnazio and Klein value data justice over data ethics, co-liberation over superficial allyship, equity over equality, and intersectionality above all else. The book offers extremely straightforward definitions for each of these terms, so much so that its content starts to come across as an Introduction to Feminist Theory 101 textbook. This is not to say that this approach makes the book any less impactful or venerable - in fact, it serves as a clear and compact framework for beginners to both feminism and data studies. D'lgnazio and Klein see their work and research as iJournal, Vol 6, No. 2 , 
a call to action. But they do not claim to have all the answers to these intricate questions, nor do they pretend that technological and algorithmic modifications have the potential to act as a panacea for systemic inequalities. Ultimately, the book promotes a symbiotic collaboration between the marginalized and the privileged — what they refer to as co-liberation and pluralism - as a comprehensive information infrastructure that responds to systems of patriarchy, colonialism, racism, and cissexism.

\section{References}

D’lgnazio, C., \& Klein, L. F. (2020). Data feminism. MIT Press. 